\title{
On Accurate Simulations of Thin-Film Solar Cells With a Thick Glass Superstrate
}

\author{
Klaus Jäger, ${ }^{1,2, *}$ Martin Hammerschmidt, ${ }^{2}$ Grit Köppel, ${ }^{1}$ \\ Sven Burger, ${ }^{2}$ and Christiane Becker ${ }^{1}$ \\ ${ }^{1}$ Young Investigator Group Nano-SIPPE, Helmholtz-Zentrum Berlin für Materialien und Energie GmbH, \\ Kekuléstraße 5, 12489 Berlin, Germany \\ ${ }^{2}$ Computational Nano Optics, Zuse Institute Berlin, Takustraße 7, 14195 Berlin, Germany \\ *klaus.jaeger@helmholtz-berlin.de
}

\begin{abstract}
The air-glass interface significantly affects the reflectivity of nanotextured layer stacks on thick glass superstrates. We estimate this effect with an a posteriori approach applied to results obtained with FEM. Further, we give experimental proofs.
\end{abstract}

OCIS codes: 000.4430 (Numerical approximation and analysis); 050.1940 (Diffraction); 310.6188 (Spectral properties)

\section{Introduction}

Numerical Maxwell solvers are well suited for optical simulations of layer stacks of thin optical films, which for example are used in thin-film solar cells. Because of the high computational cost, the maximal side lengths of unit cells used for these simulations are limited.

In practice, such thin-film stacks often are created on glass superstrates with a thickness in the order of millimetres. Hence, the superstrate cannot be fully handled by the Maxwell solvers, because in most cases the large superstrate thickness would exceed the available computational capacity. A common way to deal with this problem in the finite element method (FEM) is to represent the glass layer by a thin layer of a few hundreds of nanometres thickness, which is covered by an infinite half space filled with the same material (glass), as illustrated in Fig. 1(a). The effect of the air-glass interface then is applied to the simulation results a posteriori.

Often, the air-glass interface is taken into account with a zeroth-order correction, where only the initial reflection of the incident light beam at the air-glass interface is taken into account. Interactions of light, which is reflected from the layer stack into the glass half space, with the glass-air interface are neglected. However, if a significant fraction of this light is directed into large angles, this first-order reflection at the glass-air interface may become significant.

In this contribution we present a simple analytical method to calculate the first-order correction and we discuss the difference between the zeroth and first-order corrections in dependence of the wavelength. Further, we demonstrate the validity of this approach with experimental data. The findings presented in this contribution are important for accurately performing optical simulations of solar cells on thick glass superstrates.

\section{Theory}

In numerical simulations, periodically structured thin-film layer stacks are usually treated with periodic boundary conditions on the side faces of the unit cell. Because of this perfect periodicity, the far-field reflection and transmission into the top and bottom infinite half spaces, which cover the layer stack, only happens into discrete and well-defined channels [1]. The reflectivity $R$ of the structure in the glass halfspace can be calculated with

$$
R(\lambda)=\frac{1}{\left|\mathbf{E}_{i}^{g}(\lambda)\right|^{2} \cos \theta_{i}^{g}(\lambda)} \sum_{n}\left|\mathbf{E}_{n}^{g}(\lambda)\right|^{2} \cos \theta_{n}^{g}(\lambda),
$$

where the electromagnetic field components $\mathbf{E}_{n}^{g}$ and the angles $\theta_{n}^{g}$ are output from the Maxwell solver. The subscript $i$ denotes the incident wave and the superscript $g$ denotes fields and angles in glass, as depicted in Fig. 1(a). The sum is taken over all the channels into which the structure reflects.

In the zeroth-order correction the reflectivity $R$ is corrected with

$$
R^{0}(\lambda)=R(\lambda)\left[1-R^{g}(\lambda)\right]+R^{g}(\lambda),
$$


(a) air

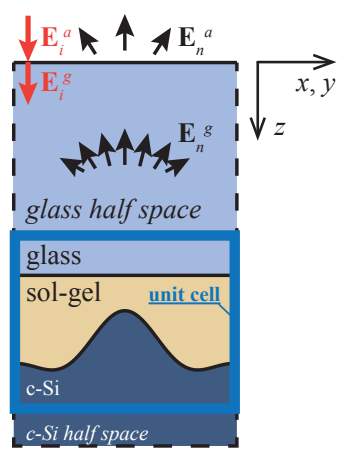

(b)

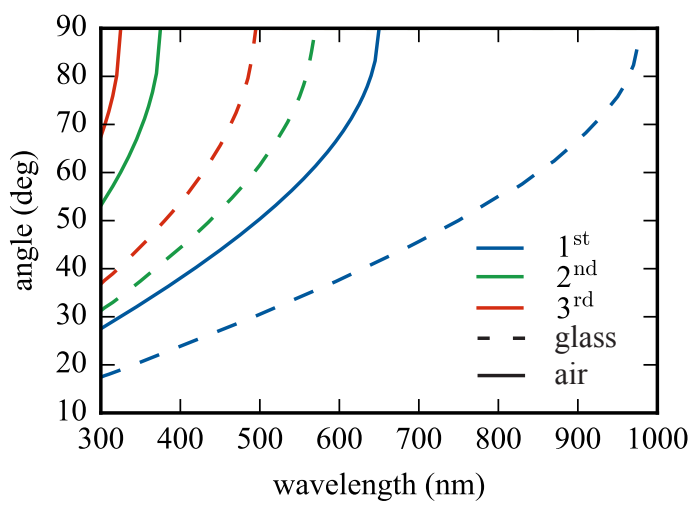

Fig. 1. (a) A cross section through a periodical unit cell with a sinusoidal interface [1] and the glass and silicon half spaces. The first-order correction described in this paper is performed for the propagation of light from glass into air. All the electric field components needed for this calculation are depicted in the sketch. (b) The angles of the diffraction orders into which light is scattered from a hexagonal periodic structure with a period of $P=750 \mathrm{~nm}$. The figure shows the angles in glass and in air. In glass, the diffraction orders are present up to much longer wavelength than in air, where above $650 \mathrm{~nm}$ only the zeroth order is present. In glass, also the $4^{\text {th }}$ and $5^{\text {th }}$ diffraction orders are present (not shown). Note that for the zeroth diffraction order we have $\theta_{g}=\theta_{a} \equiv 0$.

where the superscript 0 denotes the zeroth order correction, $R^{g}$ is the reflectivity of the air-glass interface (about $4 \%$ at normal incidence) and $\lambda$ is the wavelength $[1,2]$.

In the first-order correction also light is taken into account, which is reflected from the layer stack into the glass half space and subsequently is partially reflected back by the glass-air interface into the layer stack. For its calculation, the electric field vectors $\mathbf{E}_{n}^{a}$ and angles $\theta^{a}$ in air must be derived using the Fresnel equations and Snell's law, respectively,

$$
R^{1}=\frac{1}{\left|\mathbf{E}_{i}^{a}\right|^{2} \cos \theta_{i}^{a}} \sum_{n}\left|t_{n}^{s} \mathbf{E}_{s, n}^{g}+t_{n}^{p} \mathbf{E}_{p, n}^{g}\right|^{2} \cos \theta_{n}^{a}+R^{g} .
$$

Here, we have to decompose the $\mathbf{E}_{n}^{g}$ vectors into $s$ - and p-polarized components before multiplying them with the Fresnel coefficients $t_{n}^{s}$ and $t_{n}^{p}$, respectively. Note that these transmission coefficients describe waves that are transmitted from glass into air but that $\mathbf{E}_{i}^{a}$ and $\mathbf{E}_{i}^{g}$ are connected to each other via transmission from air into glass.

The deviation between $R^{0}$ and $R^{1}$ can be neglected, if the thin-film layer stacks only reflect specularly or when the fraction scattered into large angles is small. For example, take an optical system with $R=0.1$ that only reflects specularly: using $R^{g}=0.04$ we find $R^{0}=0.136$. The first order correction takes the light into account that is reflected back into the layer stack at the air-glass interface, $R^{1}=R^{0}-R R^{g}\left(1-R^{g}\right)$. We obtain $R R^{g}\left(1-R^{g}\right)=0.384 \%$. However, if a significant part or the light is reflected into large angles, $R^{1}$ and $R^{0}$ may differ significantly from each other.

\section{Results}

The reflected field components in glass $\mathbf{E}_{n}^{g}(\lambda)$ and the their angles $\theta_{n}^{g}(\lambda)$ with respect to the $z$-axis were obtained with the Maxwell solver JCMwave, which utilizes the finite elements method (FEM) [3].

Figure 1(b) shows the angles of the different refraction orders in glass and air for light that is refracted at an hexagonal periodic structure with $750 \mathrm{~nm}$ period. The zeroth diffraction order $\left(\theta_{g}=\theta_{a} \equiv 0\right)$ is not shown. In glass, the different diffraction orders are present until much longer wavelength than in air because of the critical angle of the air-glass interface $\left(\theta_{\text {crit }} \approx 41^{\circ}\right)$.

As a consequence, $R^{1}$ and $R^{0}$ may deviate considerably from each other, when the number of diffraction orders in glass and air differs. Figure 2 shows $1-R^{1}$ and $1-R^{0}$ for the layer stack sketched in Fig. 1(a) for a negative cosine texture that forms the interface between sol-gel and the silicon absorber [1]. Indeed, large differences are seen between $R^{1}$ and $R^{0}$. Above $970 \mathrm{~nm}$, where only the zeroth diffraction order can leave the nanotexture, the differences are very 
(a)

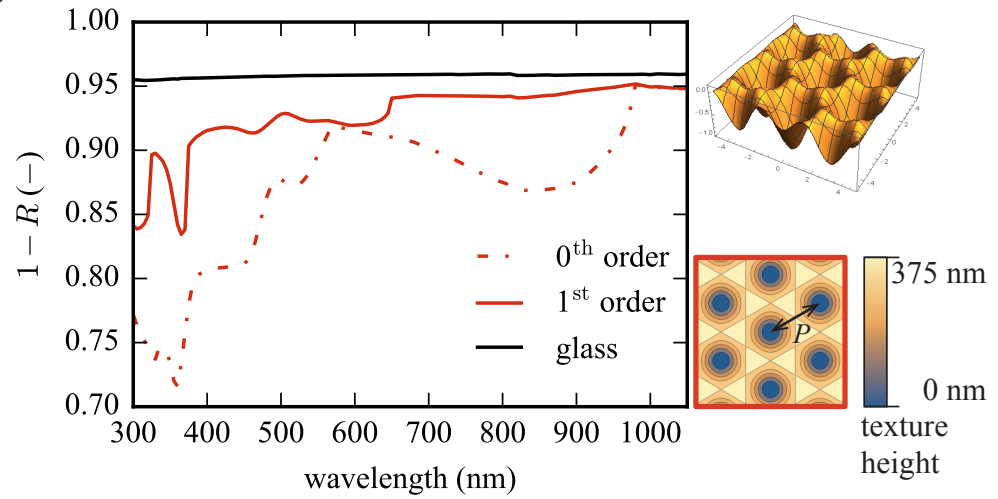

(b)

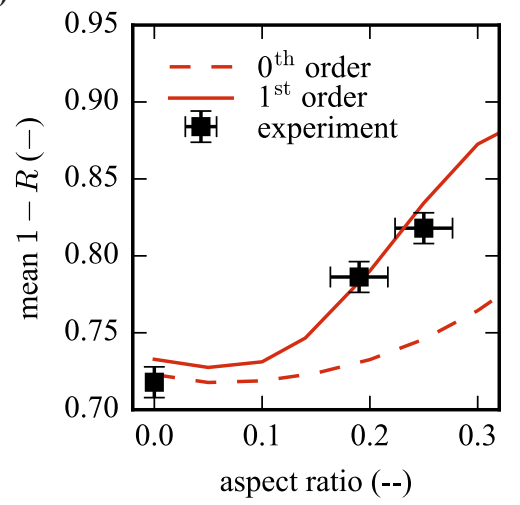

Fig. 2. (a) $1-R$ as obtained with the $0^{\text {th }}$ - and $1^{\text {st }}$-order correction for the layer stack depicted in Fig. 1(a). The results are presented for the negative cosine "-cos" geometry introduced in Ref. [1] with $P=750 \mathrm{~nm}$ period and an aspect ratio of 0.5 (structure height $375 \mathrm{~nm}$ ), which is illustrated on the right hand side. The differences between the two corrections are because of diffraction orders that are present in glass but cannot propagate into air [see Fig. 1(b)]. Above $970 \mathrm{~nm}$, where only the $0^{\text {th }}$ order is reflected from the unit cell into the glass half space, the difference between $1-R^{0}$ and $1-R^{1}$ is very small. (b) Mean $1-R$ between 400 and $600 \mathrm{~nm}$ wavelength obtained with the two corrections and for experimental data for a nanotexture period of $P=750 \mathrm{~nm}$. For the calculations, the "-cos" geometry was used.

small. Effects of the glass superstrate on the reflectivity in the range of $10 \%$ are in agreement with earlier findings by Lockau et al. [4].

Figure 2(b) shows the mean $1-R$ between 400 and $600 \mathrm{~nm}$ wavelength for the two corrections and experimental data as a function of the aspect ratio of the nanotexture and a period of $750 \mathrm{~nm}$. The experimental samples were prepared using a solid-phase crystallisation process, as described in Ref. [5]. The first-order correction resembles the experimental data much better than the zeroth-order correction.

\section{Outlook}

Reflection values obtained with the first-order correction, which is explained in this manuscript, may deviate strongly from those obtained with the zeroth order correction. The first order correction resembles experimental data much better then the zeroth order correction. Nanostructured thin-film layer stacks with a thick glass superstrate can also be handled with FEM together with a rigorous domain decomposition approach [6]. During the 2016 OSA PV meeting we also will present results obtained with that approach and compare it to results obtained with the first order correction.

\section{References}

1. K. Jäger, C. Barth, M. Hammerschmidt, S. Herrmann, S. Burger, F. Schmidt, and C. Becker, "Simulations of sinusoidal nanotextures for coupling light into c-Si thin-film solar cells," Opt. Express 24, A569-A580 (2016).

2. O. Isabella, S. Solntsev, D. Caratelli, and M. Zeman, "3-D optical modeling of thin-film silicon solar cells on diffraction gratings,” Prog. Photovolt: Res. Appl. 21, 94-108 (2013).

3. J. Pomplun, S. Burger, L. Zschiedrich, and F. Schmidt, "Adaptive finite element method for simulation of optical nano structures," Phys. Status Solidi B 244, 3419-3434 (2007). http : / j jcmwave . com.

4. D. Lockau, L. Zschiedrich, S. Burger, F. Schmidt, F. Ruske, and B. Rech, "Rigorous optical simulation of light management in crystalline silicon thin film solar cells with rough interface textures," Proc. SPIE 7933, 79330M (2011).

5. G. Köppel, B. Rech, and C. Becker, "Sinusoidal nanotextures for light management in silicon thin-film solar cells," Nanoscale 8, 8722-8728 (2016).

6. A. Schädle, L. Zschiedrich, S. Burger, R. Klose, and F. Schmidt, "Domain decomposition method for Maxwell's equations: Scattering off periodic structures,” J. Comput. Phys. 226, 477 - 493 (2007). 